$\xi_{x-1}$

\title{
Performance Comparison of Routing Protocols for Underwater Sensor Networks
}

\author{
Komal Memon, Nafeesa Bohra, Faisal K Shaikh \\ Department of Telecommunication, \\ Mehran University of Engineering and Technology Jamshoro Sindh, PakistanKorea \\ *Corresponding author E-mail: komal.memon@admin.muet.edu.pk
}

\begin{abstract}
There is a great demand of an Underwater Sensor Networks (UWSNs) in applications of water monitoring and offshore exploration. In such applications, network comprises of multiple sensor nodes which are deployed at different locations and depths of water. Sensor nodes perform collective tasks such as data collection and data transmission to other nodes or Base Station (BS). The bottom nodes are located at depth of water, and are not able to communicate directly with the surface level nodes, these nodes require multi-hop communication with appropriate routing protocol. Therefore, an energy efficient routing protocols are used for such scenarios, which is necessary as well as challenging task. As sensors are battery operated devices, which are really problematic to recharge or replace. The error and propagation path delays are high in acoustic channels therefore underwater communication is much effected. Realizing the circumstances, more attention has been given to compare energy efficient routing protocols which comparatively consume low energy and achieve high throughput. This paper, comprises of analysis and comparison of existing UWSN based efficient energy routing protocols. Based upon the analysis and comparison, VBF and DBR have been proposed that fulfill the requirements. The analysis is done on NS-2 and for comparison, the performance metrics which are evaluated are: Packet delivery Ratio (PDR), energy consumption, throughput and average End to End (E2E) delay. The results show that VBF protocol consume very large amount of energy as compared to DBR protocol. Whereas DBR protocol have characteristics like low energy consumption, minimum delay high PDR and high throughput than VBF protocol.
\end{abstract}

Keywords: Energy efficient; Routing, VBF; DBR, Underwater sensor networks.

\section{Introduction}

Almost 75 percent of earth is covered by deep oceans and sea. These harsh environments doesn't allow human to explore environmental monitoring easily. The existing sensing technologies does not fulfill the requirements of low-cost equipment and easy deployment [1]. As there are very limited marine resources to monitor the changes in water, therefore it should be effectively monitored to conserve these resources.

In last few decades lot of attention has been paid to marine research, UWSN have served as a platform in this domain. UWSN have great demand in multiple applications like disaster prevention, gas and oil exploration, security and safety, to surveillance [2]. UWSN is the ideal platform for such type of applications. Acoustic communications are affected by multipath, propagation delay, noise, Doppler spread and path loss [3]. These all parameters creates variation in spatial and temporal aspects of acoustic channel, and bandwidth of acoustic channel becomes too limited [3]. Further communication range of acoustic channel is also reduced in comparison of radio channel. For these reasons there is need of efficient routing for data transmission in underwater communication. In 3-D area of sensor networks when we implements energy efficient routing protocols, sensor nodes can efficiently monitor environmental events. In under water communication radio signals does not work fine therefore UWSNs have to use acoustic Signals, as shown in figure 1.

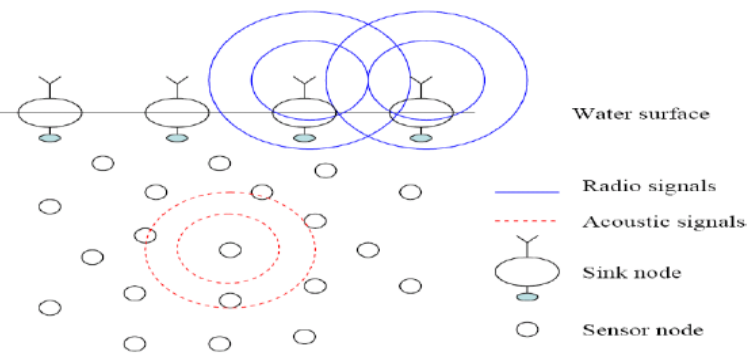

Figure 1: General scenario of data transmission in UWSN [4]

As acoustic channels have features such as high error, large latency, and low bandwidth, therefore efficient routing is needed. Another reason is nodes are battery operated devices, which is quite difficult task to replace or recharge the batteries. It is necessary as well as challenging to implement efficient routing protocols for such type of networks. 
The proposed idea introduces the concept of environmental monitoring using energy efficient routing protocols and allow us to use techniques that can be used to minimize energy consumption in underwater communication system. The paper is segmented in following major parts: section I, introduces the concept, whereas the literature work is described in section II, while section III contains the, brief description of energy efficient routing protocols for UWSN, whereas section IV consists of simulation and results while section V concludes the paper and give future directions.

\section{Related Work}

In UWSN major concern is energy saving of sensor nodes because they are battery powered devices. In aquatic medium required power for data transmission is about more than 100 times the required power for data reception [5]. Therefore significant issue in research domain is to design energy-efficient, scalable and robust routing protocols.

There are multiple protocols available for terrestrial network for instance LEACH (Low-Energy Adaptive Clustering Hierarchy) [6], GEAR (Geographic and Energy Aware Routing) [7], Rumor routing [8], Directed Diffusion [9], these all are not suitable to UWSN due to limitation of acoustic channel in underwater medium. In literature, many efforts are taken to simulate propagation models of acoustic networks [10].Some of simulators like: NS-2 and OPNET are widely used for terrestrial networks, but not for UWSN. There are multiple reasons for which acoustic propagation models could not simulated on these simulators, such as acoustic signal have very slow speed in water, secondly, attenuation model of acoustic signal is different than radio signal, thirdly UWSN are generally installed in 3D, whereas the UWSN simulators support just 2D deployment. Thus in this research we have used Aqua-Sim simulator, designed for modeling of UWSN. Although Aqua-sim is also designed on the NS-2 platform. By using Aqua-Sim simulator acoustic signal attenuation model can effectively simulated. Further, it supports threedimensional network deployment. Moreover, existing codes of NS-2 can be easily integrated with Aqua-Sim. At present, three routing protocols are implemented in Aqua-Sim which are: Vector based routing protocol (VBF) [11], Depth-base Routing protocol (DBR) [12] and Q-learning-based Routing protocol (QELAR) [13]. In this paper we have focuses on analysis and comparison of VBF and DBR protocol as in literature these protocols are commonly used in underwater communication.

\section{Description of Routing Protocols}

In Aqua-Sim simulator three protocols are implemented at routing layer: VBF, DBR and QELAR as shown in figure 2.

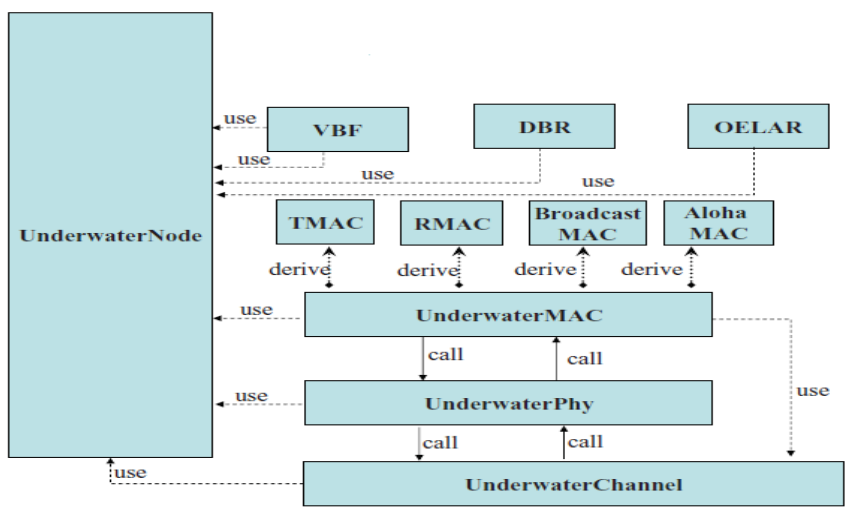

Figure 2: Aqua-Sim Classification [4]
Our research is based on analysis and comparison of energy consumption in VBF and DBR protocol. The description of these routing protocols are given as follows:

Vector-Based Forwarding (VBF) Protocol:

In this protocol each sensor node is aware about its position in network therefore this protocol is known as geographic routing protocol. Whereas in this protocol forwarding vector specifies the route from source to target. The header of each packet contains information of source, forwarder and target. In VBF when data packet is received by node, it starts calculating its own distance towards forwarder. If the measured distance of node is less than a radius which is already defined in header by vector pipe, then node will be able to forward packet other otherwise it will be discarded and search for another node [11]. In this protocol forwarding pipe is a virtual path or pipe which gives direction of path form source to target as shown in figure 3:

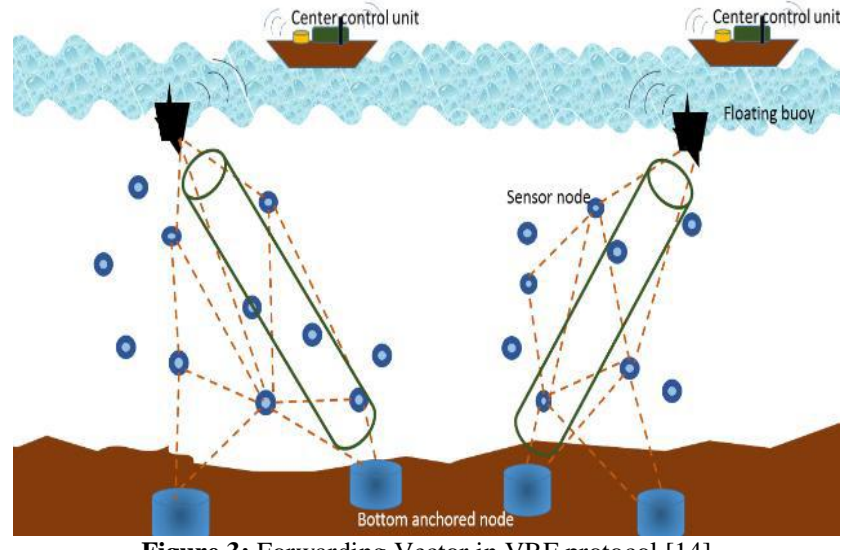

Figure 3: Forwarding Vector in VBF protocol [14]

Depth-Based Routing (DBR) Protocol:

Depth Based Routing (DBR) protocol is greedy forwarding protocol which uses depth information [12]. In UWSN sensor nodes uses depth information of deployed nodes in water and forward packets from a source to sink node as shown in figure 4. At every routing step packet follows depth reducing rule to select forwarding nodes to water surface. When packet is received in DBR, a depth information of previous node is compared with current node which is available in the packet. If receiving node is nearer to water surface it will be capable to forward packet and node will be selected for data forwarding purpose. The condition which gives the information of closer node is the depth of receiving node which should be smaller than previous hop or node. Otherwise packet is simply discarded.

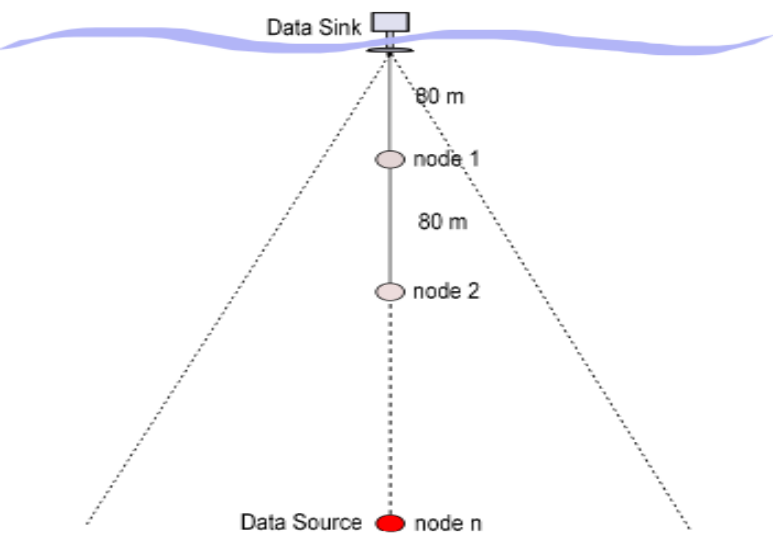

Figure 4: DBR protocol topology [12] 


\section{Simulation Scenario and Results}

This research is consists of, comparison of different performance metrics of VBF with DBR protocol. In this simulation, 20,40,60,80 and 100 nodes which are randomly organized in a space of $1000 \mathrm{~m} \times$ $500 \mathrm{~m} \times 100 \mathrm{~m} 3$-D space. At the surface one stationary sink node is deployed. While the sensor nodes are mobile which follows the random pattern of mobility. Every node have random direction and random speed to move to the new position. At every 10 seconds one packet is generated by source node, every packet have fixed size of 50 bytes. The transmission range is 120 meters in every direction. All these communication parameters are summarized in Table 1. Further at MAC layer, "Broadcast MAC" protocol is used.

Table 1: Simulation Parameters

\begin{tabular}{|c|c|}
\hline Software & Aqua-sim version 2.30 \\
\hline Topology Area & $1000 \mathrm{~m} \times 500 \mathrm{~m} \times 100 \mathrm{~m}$ \\
\hline Nodes & $20,40,60,80,100$ \\
\hline Transmission range & 120 meters \\
\hline Width of pipe & 50 meters \\
\hline Packet Size & 50 bytes \\
\hline Simulation time & 500 Seconds \\
\hline Initial Energy & 10,000 \\
\hline
\end{tabular}

In simulation, we analyzed effect of node density on PDR, delay, throughput and energy consumption in two various routing protocols, such as DBR and VBF. We change number of deployed nodes to vary node density from 20 to 100 . The comparison of these parame- ters such as PDR, end to end delay, energy consumption and throughput in VBF and DBR are plotted in Figure 5, Figure 6, Figure 7 and Figure 8 respectively.

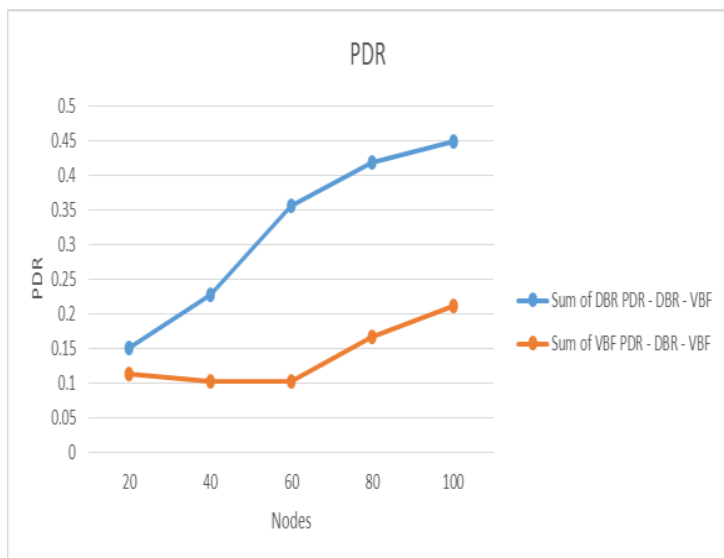

Figure 5: Packet Delivery Ratio (PDR)

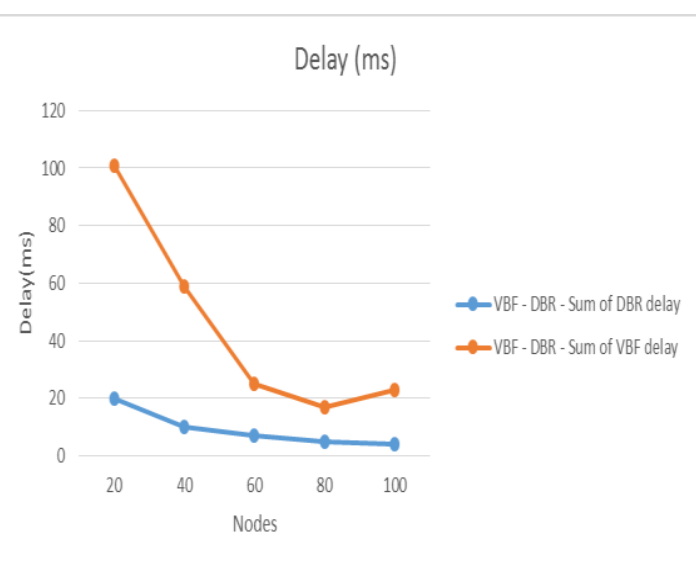

Figure 6: End to End delay (E2E)

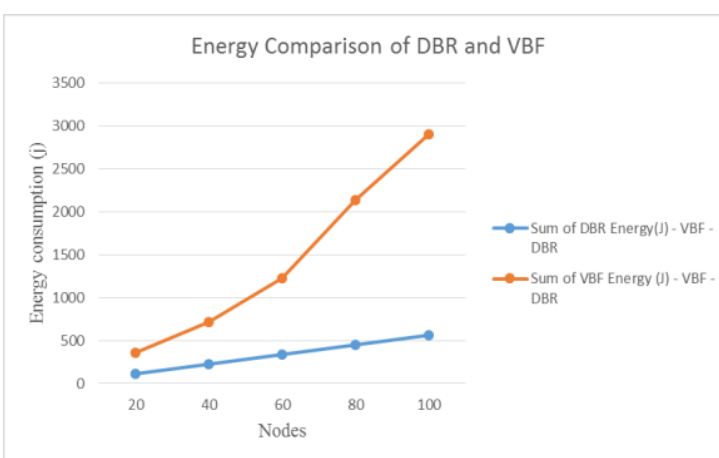

Figure 7: Energy consumption

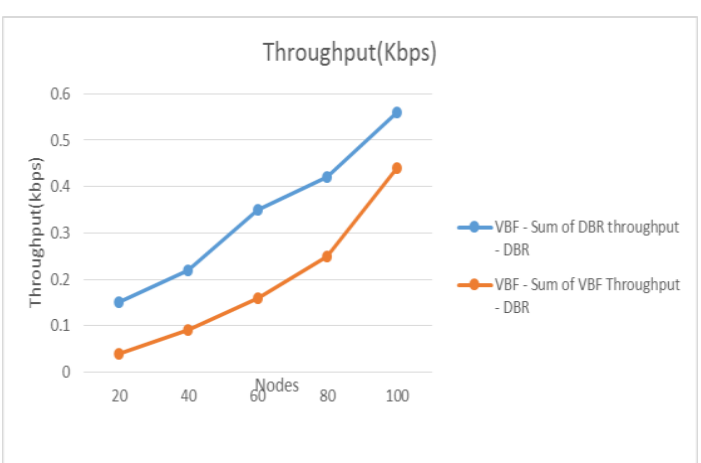

Figure 8: Throughput
The performance of these protocols have been compared with respect to node density, which is with random nodes. As results shows that in sparse networks (With lower number of nodes) energy consumption is very low as compared to dense networks. Another fact which have been observed is, VBF protocol consume very large amount of energy as compared to DBR protocol. Whereas DBR protocol have characteristics like low energy consumption, minimum delay, high PDR and high throughput than VBF protocol. These outcomes are summarized in Table 2:

Table 2: Performance metrics comparison

\begin{tabular}{|c|c|c|c|c|c|c|}
\hline Routing Protocol & PDR & Delay & Energy Efficiency & Throughput & Reliability & Performance \\
\hline VBF & Low & High & Low & Low & Low & Low \\
\hline DBR & High & Low & High & Fair & High & High \\
\hline
\end{tabular}




\section{Conclusion and Future Work}

In UWSN, when we analyze the VBF and DBR protocols performance, the results signifies that the DBR is highly recommended protocol for these networks. DBR protocol have characteristics like low energy consumption, minimum delay high PDR and high throughput as compared to VBF. It is more reliable protocol to use in UWSN. As simulation results are taken on single sink. Whereas DBR protocol may have even better performance using multiple-sink. In future work these protocols may be implemented in real time networks. We can replace one sink of DBR protocol into multiple sink networks.

\section{Acknowledgements}

This research has been carried out at the Dept. of Telecommunication Engg. Mehran UET, Jamshoro, Pakistan.

\section{References}

[1] Bayrakdar, Y., Meratnia, N., \& Kantarci, A. (2011, June). A comparative view of routing protocols for underwater wireless sensor networks. In OCEANS, 2011 IEEE-Spain (pp. 1-5). IEEE.

[2] Guo, Z., Colombi, G., Wang, B., Cui, J. H., Maggiorini, D., \& Rossi, G. P. (2008, January). Adaptive routing in underwater delay/disruption tolerant sensor networks. In Wireless on Demand Network Systems and Services, 2008. WONS 2008. Fifth Annual Conference on (pp. 31-39). IEEE.

[3] Jain, S., Pilli, E. S., Govil, M. C., \& Rao, D. V. (2015, February). Performance evaluation of congestion-aware routing protocols for underwater sensor networks with multimedia data. In Underwater Technology (UT), 2015 IEEE (pp. 1-6). IEEE.

[4] Giantsis, C., \& Economides, A. A. (2011). Comparison of routing protocols for underwater sensor networks: a survey. International Journal of Communication Networks and Distributed Systems, 7(3-4), 192-228.

[5] J. Partan, J. Kurose, B.N. Levine, A survey of practical issues in underwater networks, in Proceedings of ACM International Workshop on Underwater Networks (WUWNet), September 2006, pp. $17-24$.

[6] W. Heinzelman, A. Chandrakasan, H. Balakrishnan, An Application-Specific Protocol Architecture for Wireless Microsensor Networks, IEEE Transactions on Wireless Communications 1 (4) (2002) 660-670.

[7] Y. Yu, D. Estrin, R. Govindan, Geographical and energy-aware routing: a recursive data dissemination protocol for wireless sensor networks, UCLA Comp. Sci. Dept. tech. rep., UCLA-CSD TR010023, May 2001.

[8] D. Braginsky, D. Estrin, Rumor routing algorithm for sensor networks, in: International Conference on Distributed Computing Systems, November 2001.

[9] C. Intanagonwiwat, R. Govindan, D. Estrin, Directed diffusion: ascalable and robust communication paradigm for sensor networks, in: Proceedings of ACM MobiCom 2000, Boston, MA, 2000, pp. 56-67.

[10] W. Heinzelman, A. Chandrakasan, H. Balakrishnan, An Application-Specific Protocol Architecture for Wireless Microsensor Networks, IEEE Transactions on Wireless Communications 1 (4) (2002) 660-670

[11] P. Xie, J.-H. Cui, and L. Lao. VBF: Vector-Based Forwarding Protocolfor Underwater Sensor Networks. In Proceedings of IFIP Networking' 06, Coimbra, Portugal, May 2006. 